\title{
Clinical outcome and risk factors for complications after pulmonary segmentectomy by video-assisted thoracoscopic surgery: results of an initial experience
}

\author{
Benoît Bédat $^{1 \#}$, Etienne Abdelnour-Berchtold ${ }^{2 \#}$, Thorsten Krueger ${ }^{2}$, Jean Yannis Perentes ${ }^{2}$, Hans-Beat \\ Ris $^{2}$, Frédéric Triponez ${ }^{1}$, Marc-Joseph Licker ${ }^{3}$, Wolfram Karenovics ${ }^{1 \#}$, Michel Gonzalez ${ }^{2 \#}$ \\ ${ }^{1}$ Service of Thoracic Surgery, Hopitaux Universitaires de Genève, Genève, Switzerland; ${ }^{2}$ Service of Thoracic Surgery, Centre Hospitalier \\ Universitaire Vaudois, Lausanne, Switzerland; ${ }^{3}$ Service of Anesthesiology, Hopitaux Universitaires de Genève, Genève, Switzerland \\ Contributions: (I) Conception and design: B Bédat, E Abdelnour-Berchtold, W Karenovics, M Gonzalez; (II) Administrative support: B Bédat, E \\ Abdelnour-Berchtold, T Krueger, F Triponez, M Gonzalez; (III) Provision of study materials or patients: B Bédat, E Abdelnour-Berchtold, W \\ Karenovics, M Gonzalez; (IV) Collection and assembly of data: B Bédat, E Abdelnour-Berchtold; (V) Data analysis and interpretation: B Bédat, E \\ Abdelnour-Berchtold, W Karenovics, M Gonzalez; (VI) Manuscript writing: All authors; (VII) Final approval of manuscript: All authors. \\ \#These authors contributed equally to this work. \\ Correspondence to: Michel Gonzalez, MD. Division of Thoracic Surgery, Centre Hospitalier Universitaire Vaudois, 1011 Lausanne, Switzerland. \\ Email: michel.gonzalez@chuv.ch.
}

Background: Pulmonary anatomical segmentectomies are increasingly being done via video-assisted thoracoscopic surgery (VATS). We analyzed clinical outcomes and risk factors for post-operative complications after pulmonary segmentectomy by VATS was introduced in two institutions.

Methods: We retrospectively reviewed records of all patients who underwent anatomical pulmonary segmentectomy by VATS from 2014 to 2016 at the university hospitals of Geneva and Lausanne in Switzerland.

Results: One hundred twenty-nine patients (64 men; median age 68 years, range, 29-85 years) underwent anatomical VATS segmentectomy for primary lung tumors $(n=100)$, metastases $(n=16)$ and benign lesions $(\mathrm{n}=13)$. The overall 30 -day mortality and morbidity rates were $0.8 \%$ and $31 \%$, respectively. The reoperation rate was 4.7\% [indications: hemothorax 2, prolonged air leak (PAL) 2, segmental torsion 1, empyema 1]. Chest drainage lasted for a median of 2 days (range, 1-33 days) and patients were discharged from the hospital after a median of 6 days (range, 2-37 days). Postoperative complications were mainly associated with chronic obstructive pulmonary disease (COPD) [odds ratio (OR) 2.54 and $95 \%$ confidence interval (95\% CI), 1.18-5.47], and smoking pack-years $>50$ units (OR 5.27; 95\% CI, 1.68-16.55). Nine patients (9\%) presented with distant recurrences. Nodule size $>2 \mathrm{~cm}$ was associated with decreased disease-free survival (DFS) ( $\mathrm{P}=0.04)$. There was no association between surgical experience in VATS segmentectomy and DFS or postoperative complications.

Conclusions: Segmentectomies can be safely performed by VATS in an initial experience and result in favorable clinical outcome. COPD and smoking pack-years are associated with an increased risk of complications.

Keywords: Pulmonary segmentectomy; thoracoscopy; lung cancer; complications

Submitted Jan 24, 2018. Accepted for publication Jun 24, 2018.

doi: $10.21037 /$ jtd.2018.07.42

View this article at: http://dx.doi.org/10.21037/jtd.2018.07.42 


\section{Introduction}

Pulmonary anatomical segmentectomy is a parenchymasparing technique that is increasingly proposed for benign lesions, metastases, or for patients with non-small cell lung cancer (NSCLC) who cannot tolerate lobectomy. Segmentectomies can be technically challenging, but are being proposed more frequently through the use of video-assisted thoracoscopic surgery (VATS). Moreover, for early stage NSCLC, pulmonary segmentectomy has shown promising results and is currently considered safe for early stage tumors $<2 \mathrm{~cm}$ in an appropriate location and with sufficient resection margins (1-5). So far, despite its attractiveness, VATS segmentectomy remains technically arduous. Furthermore, although the impact of surgeon volume on outcomes for lung surgery by VATS is significant (6-7), the impact of surgeon volume on outcomes after pulmonary segmentectomy specifically is not known. Therefore, we undertook a cohort study to analyze the clinical outcomes and identify risk factors for post-operative complications after pulmonary segmentectomy by VATS was introduced in two university hospitals.

\section{Methods}

\section{Patients}

We retrospectively reviewed the records of all patients who underwent anatomical pulmonary segmentectomies by VATS from January 2014 to December 2016 at the university hospitals of Geneva and Lausanne in Switzerland. The Local Ethics Committee approved the study and individual consent was waived (No. 2017-01767). We excluded patients whose procedure required conversion to a thoracotomy and patients who had a completion lobectomy in cases of incomplete resection or lymph node involvement on frozen section. Three surgeons who had done $>100$ cases of VATS lobectomy performed all segmentectomies in the two centers. All patients with NSCLC had extensive positron emission tomography-computed tomography (PET-CT) imaging to exclude lymph node involvement. After presentation at an interdisciplinary tumor board, the indication to proceed to VATS segmentectomy was proposed in patients with solitary nodule of less than $2 \mathrm{~cm}$ peripherally located in a specific segment. In patients unfit to benefit a lobectomy, segmentectomy was proposed even for lesion of more than $2 \mathrm{~cm}$. All patient had PET-CT excluding hilar or mediastinal lymph node extension.

\section{Surgical technique}

VATS segmentectomy was performed under general anesthesia with lung isolation by double lumen intubation. Surgical resection was undertaken using a standardized three-port approach (utility incision in the fourth intercostal space, one for $10-\mathrm{mm} 30^{\circ}$ thoracoscope in the seventh intercostal space anteriorly and one posteriorly). Segmentectomy was performed with individual dissection of the segmental bronchus, arteries and veins. All bronchovascular structures were transected using endoscopic staplers or energy device. In cases of NSCLC, dissection of hilar and mediastinal lymph nodes was complete. Frozen section was performed for suspected hilar lymph nodes and completion lobectomy undertaken in cases of lymph node involvement. All surgical specimens were extracted in a protective bag to prevent chest wall seeding of malignant disease. The intersegmental plane was divided using staplers. If available, indocyanine green fluorescence was used to identify the intersegmental plane as previously described by Guigard et al. (8).

\section{Measurements and outcomes}

Individual medical records were extracted from the hospital data management system. All records were analyzed with respect to patient demographics, histologic findings of the lesion, size and localization of the lesion, type of segmentectomy, disease-free interval, localization of recurrence, surgical outcome, and post-operative morbidity and mortality. The experience of surgeons in VATS segmentectomy is a continuous variable and defined as the number of interventions done over the course of the study.

The primary endpoint was defined as any cardiopulmonary complications with TMM grades $\geq 2$ as reported previously, and included the following: atrial fibrillation, myocardial infraction, pneumonia, pneumothorax, hemothorax, prolonged air leak (PAL) and acute respiratory distress syndrome (ARDS) (9). Pneumonia was defined by the need for antibiotics for a suspected respiratory infection according to changed lung opacities, fever, or an elevated white blood cell count $>12,000$ per mcL. PAL was defined as an air leak lasting beyond postoperative day 7. ARDS was diagnosed according to the Berlin definition based on Ferguson et al. (10). For lung cancer, the $7^{\text {th }}$ edition of TNM classification was used. 
Table 1 Preoperative characteristics of patients undergoing segmentectomy by video-assisted thoracoscopic surgery

\begin{tabular}{|c|c|}
\hline Characteristics & Number \\
\hline Patients & 129 \\
\hline \multicolumn{2}{|l|}{ Gender, n [\%] } \\
\hline Female & $65[50]$ \\
\hline Male & $64[50]$ \\
\hline Median age (years) & 68 (range, 29-85) \\
\hline \multicolumn{2}{|l|}{ Comorbidities, n [\%] } \\
\hline COPD & $48[37]$ \\
\hline History of cancer & 49 [38] \\
\hline Current smoker & $65[50]$ \\
\hline SPY $>50$ & $33[26]$ \\
\hline \multicolumn{2}{|l|}{ ASA, $n$ [\%] } \\
\hline I & $1[1]$ \\
\hline II & $81[63]$ \\
\hline III & $43[33]$ \\
\hline IV & $4[3]$ \\
\hline Predicted FEV $1, \%$ (SD) & $87[21]$ \\
\hline Predicted DLCO, \% (SD) & 77 [19] \\
\hline \multicolumn{2}{|l|}{ Indications, n [\%] } \\
\hline Suspect SPN & $95[74]$ \\
\hline NSCLC $<2 \mathrm{~cm}$ & $21[16]$ \\
\hline Metastasis & $8[6]$ \\
\hline Benign tumor & $5[4]$ \\
\hline
\end{tabular}

ASA, American Society of Anesthesiology Physical status; COPD, chronic obstructive pulmonary disease; DLCO, diffusing lung capacity for carbon monoxide; $F_{E V}$, forced expiratory volume in one second; NSLC, non-small cell lung cancer; SPN, solitary pulmonary nodule; SPY, smoking pack years.

\section{Statistical analysis}

Continuous variables are presented as mean or median as appropriate and categorical variables are expressed as percentages. Univariate logistic regression analyses were performed to determine the association between patient characteristics and the presence of complications. For continuous variables, the assumption of linearity has been met. Operation time and experience of surgeons in VATS segmentectomy were evaluated using symmetric regression procedures. Patients with metastasis or benign tumors were excluded from the time-to-event analysis. The event was considered to be any recurrence in order to obtain diseasefree survival (DFS) at 3 years. Patients were censored at the time of their last follow-up. Kaplan-Meier estimates were assessed for the nodule size, the type of histology and the surgical experience. For this analysis, the surgical experience was categorized in three groups because the linearity has not been met. Log-rank tests were used to compare differences in Kaplan-Meier estimates. All analyses were performed using STATA software, version 14 (StataCorp LLC, Texas, USA).

\section{Results}

\section{Patient characteristics}

Between January 2014 and December 2016, 129 patients (64 men, 65 females) with a median age of 68 years (range, 29-85 years) underwent anatomical pulmonary segmentectomy by VATS (Table 1). Forty-eight (37\%) patients had chronic obstructive pulmonary disease (COPD), defined by a post-bronchodilator forced expiratory volume in one second $\left(\mathrm{FEV}_{1}\right) /$ forced vital capacity $<0.7$. Fifty percent were current smokers; median smoking pack-years was 30 (IQR, 0-50). The $\mathrm{FEV}_{1}$ and the diffusing lung capacity for carbon monoxide (DLCO) were, respectively, $87 \% \pm 21 \%$ and $77 \% \pm 19 \%$ of the predicted value. The presence of a suspect solitary pulmonary nodule was the major indication for segmentectomy (74\%), followed by proven primary lung tumors (16\%), metastases (6\%) and benign lung tumors (4\%). The segmentectomies were predominantly performed for upper lobe diseases (79 segmentectomies), followed by lower lobe processes (60 segmentectomies) (Table 2). In 5 patients, two segmentectomies were performed in two different lobes. The intersegmental plane was identified with indocyanine green fluorescence imaging $33 \%$ of the patients.

\section{Postoperative morbidity and mortality}

During the study period, 3 patients had thoracotomy conversion for calcified hilar lymph node $(\mathrm{n}=2)$ and bleeding of the pulmonary vein $(n=1)$. Three additional patients had completion lobectomy for metastatic hilar lymph node $(n=2)$ or incomplete margin resection of the intersegmental plane $(n=1)$. The mean operation time was $151 \pm 121$ minutes and was not correlated with surgeon experience $(r=0.11, \mathrm{P}=0.2)$. 
Table 2 Localization and types of segmentectomies performed in 129 patients

\begin{tabular}{lc}
\hline Segmentectomy & Right/left side (N) \\
\hline Number of patients & $49 / 80$ \\
Number of segmentectomies & $56 / 83$ \\
Upper lobe segmentectomies & $25 / 54$ \\
Segment 1 & $8 / 2$ \\
Segment 2 & $5 / 4$ \\
Segment 3 & $3 / 5$ \\
Segment 1-2 & $6 / 13$ \\
Segment 1-3 & $2 / 2$ \\
Segment 1-2-3 & $0 / 20$ \\
Segment 4-5 & $0 / 8$ \\
Segment 5 & $1 / 0$ \\
Lower lobe segmentectomies & $31 / 29$ \\
Segment 6 & $15 / 20$ \\
Segment 8 & $2 / 2$ \\
Segment 9 & $4 / 1$ \\
Segment 10 & $4 / 2$ \\
Basilar segments & $6 / 4$ \\
Multiple lobes & $2 / 3$ \\
\hline &
\end{tabular}

The overall 30 -day mortality and morbidity were $0.8 \%$ and $31 \%$, respectively. The patient who died did so on post-operative day 15 from sepsis and ARDS. In terms of morbidity (Table 3), bronchopneumonia was the most frequent complication (17\%), followed by PAL (10\%). Six (4.7\%) patients required reoperation: for thoracic bleeding in two patients, PALs in two, and segmental torsion and empyema in one patient, respectively. The median duration of thoracic drainage was 2 days (IQR, 1-4 days) and the median length of hospitalization was 6 days (IQR, 4-8 days) (Table 3).

On univariate analysis, COPD (OR 2.54, 95\% CI, $1.18-5.47)$ and smoking pack-years $>50$ units (OR 5.27, 95\% CI, 1.68-16.55) were significantly associated with postoperative complications (Table 4). There was a strong correlation between the presence of COPD, active smoking and smoking pack-years $>50(\mathrm{P}<0.001)$. Conversely, age, gender, $\mathrm{FEV}_{1}$, DLCO, ASA score and type of segmentectomy were not associated with a higher risk of
Table 3 Clinical outcome in 129 patients who underwent segmentectomy by video-assisted thoracoscopic surgery

\begin{tabular}{|c|c|}
\hline Variables & Number \\
\hline Drain removal (day), median [IQR] & $2[1-4]$ \\
\hline LOS (day), median [IQR] & $6[4-8]$ \\
\hline Morbidity, n [\%] & 40 [31] \\
\hline Bronchopneumonia & $22[17]$ \\
\hline Pulmonary air leak & $13[10]$ \\
\hline Atrial fibrillation & $7[5]$ \\
\hline ARDS & $4[3]$ \\
\hline Hemothorax & $2[1]$ \\
\hline Primary lung cancer, n [\%] & $100[78]$ \\
\hline Adenocarcinoma & $78 / 100[78]$ \\
\hline Squamous cell carcinoma & $15 / 100[15]$ \\
\hline NET & $7 / 100[7]$ \\
\hline Tumor size, mean [range] & $17[6-48]$ \\
\hline \multicolumn{2}{|l|}{ TNM classification, n [\%] } \\
\hline pT1 & $80 / 100[80]$ \\
\hline pT2 & $17 / 100[17]$ \\
\hline pT3 & $1 / 100[1]$ \\
\hline pT4 & $2 / 100[2]$ \\
\hline $\mathrm{pNO}$ & $92 / 100[92]$ \\
\hline $\mathrm{pN} 1$ & $1 / 100[1]$ \\
\hline $\mathrm{pN} 2$ & $7 / 100[7]$ \\
\hline \multicolumn{2}{|l|}{ Stage $\left(7^{\text {th }}\right.$ edition), $\mathrm{n}[\%]$} \\
\hline 1 & $90 / 100[90]$ \\
\hline II & $3 / 100[3]$ \\
\hline III & $7 / 100[7]$ \\
\hline
\end{tabular}

LOS, length of hospital stay; ARDS, acute respiratory distress syndrome; NET, neuroendocrine tumor.

major complications. Furthermore, there was no association between complication rate and surgical experience in VATS segmentectomy $(\mathrm{P}=0.4)$.

\section{Oncological outcome}

Histological analysis revealed a primary lung tumor in 100 patients (78\%), metastases in 16 patients $(12 \%)$, and benign lesions in $13(10 \%)$. Of the primary lung tumors, $78 \%$ were 
Table 4 Risk factors for complication after VATS segmentectomy

\begin{tabular}{|c|c|c|}
\hline \multirow[b]{2}{*}{ Variable } & \multicolumn{2}{|c|}{ Complications } \\
\hline & $\begin{array}{l}\text { Odds ratio } \\
(95 \% \mathrm{Cl})\end{array}$ & $P$ value \\
\hline Age, each 1-year increase & $1.02(0.98-1.07)$ & 0.24 \\
\hline Female vs. male & $1.13(0.54-2.38)$ & 0.75 \\
\hline COPD (yes vs. no) & $2.54(1.18-5.47)$ & 0.02 \\
\hline $\mathrm{FEV}_{1}(\geq 80 \%$ vs. $<80 \%)$ & $1.91(0.88-4.16)$ & 0.1 \\
\hline DLCO ( $\geq 80 \%$ vs. $<80 \%)$ & $1.59(0.73-3.44)$ & 0.24 \\
\hline Current smoking (yes vs. no) & $2.38(0.1-5.16)$ & 0.03 \\
\hline \multicolumn{3}{|l|}{ SPY } \\
\hline $1-10$ & $1.55(0.14-16.85)$ & 0.72 \\
\hline $11-49$ & $2.86(0.89-9.18)$ & 0.08 \\
\hline $50-120$ & $5.27(1.68-16.55)$ & $<0.01$ \\
\hline ASA score (3 vs. 1) & $2.37(0.23-24.7)$ & 0.47 \\
\hline Segment (inferior vs. superior lobe) & $0.61(0.76-3.43)$ & 0.22 \\
\hline Experience, each 1 patient more & 1.00 & 0.4 \\
\hline
\end{tabular}

ASA, American Society of Anesthesiology Physical status; COPD, chronic obstructive pulmonary disease; DLCO, diffusing lung capacity for carbon monoxide; $\mathrm{FEV}_{1}$, forced expiratory volume in one second; SPY, smocking pack years.

adenocarcinoma, $15 \%$ were squamous cell carcinoma, and $7 \%$ were neuro-endocrine tumors. The mean tumor size was $17 \mathrm{~mm}$ (range, 6-48 mm). Eighty patients had pT1 tumors, 17 had pT2, 1 had pT3 and 2 had pT4 tumors. A tumor $<2 \mathrm{~cm}$ was found in $79 \%$ of patients. In patients undergoing mediastinal lymph node dissection, the mean number of extracted lymph nodes was $11.8 \pm 7.9$. Nodal upstaging was found in eight patients (8\%) including one $\mathrm{pN} 1$ and seven $\mathrm{pN} 2$ (Table 3).

The median follow-up time was 19 months (IQR, 13-22 months), and no patient was found to have a local recurrence in the operated lobe. However, nine (9\%) patients did have recurrences, including four tumors in another lobe, one regional lymph node recurrence and four distant recurrences. Kaplan-Meier DFS curves at three years are shown in Figure 1. A nodule size $>2 \mathrm{~cm}$ was associated with decreased DFS $(\mathrm{P}=0.04)$. Log rank analyses showed no association between the DFS and surgical experience with VATS segmentectomy, type of histology, or nodule staging.

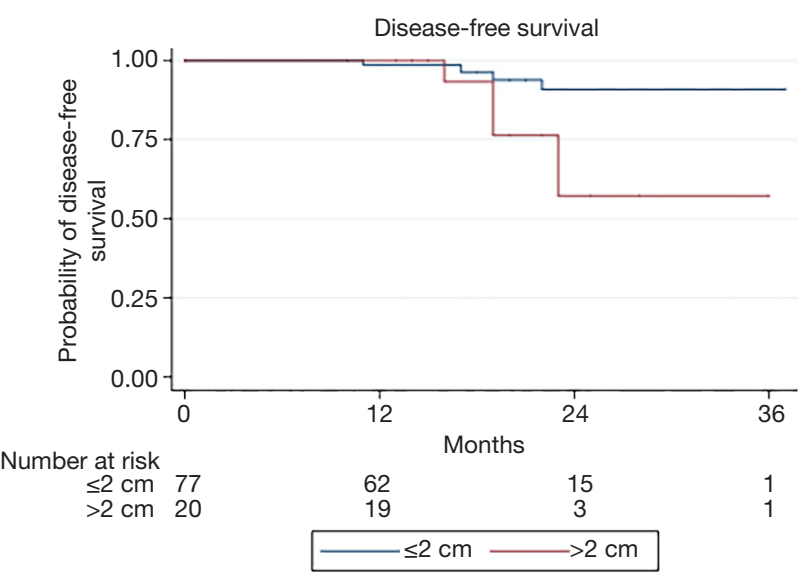

Figure 1 Kaplan-Meier curves of disease-free survival for nodule $\leq 2 \mathrm{~cm}$ (blue) versus nodule $>2 \mathrm{~cm}$ (red) $(\mathrm{P}=0.04)$.

\section{Conclusions}

Pulmonary anatomical segmentectomy is increasingly being proposed for benign and malignant lesions. However, this surgical technique is demanding and few studies have reported outcomes when VATS is used. Our results show that segmentectomy by VATS can be realized safely with acceptable morbidity and low mortality even during an initial experience.

VATS segmentectomy has been proposed as a parenchyma-sparing procedure and should result in better postoperative pulmonary function and a faster postoperative recovery than VATS lobectomy. However, these benefits are not clearly demonstrated in the literature. The main reasons for this are that pulmonary function test results are difficult to interpret and VATS and thoracotomy procedures are lumped together (11-14).

Because VATS segmentectomy is technically difficult and its beneficial effect on the postoperative course has not yet been demonstrated, it is important to analyze complications, especially early in the use of this procedure. Unfortunately, VATS and thoracotomy procedures are also lumped together in many studies $(15,16)$. However, one study reported a greater incidence of air leak and pulmonary complications after VATS segmentectomy than VATS lobectomy, although the sample size was only 35 patients (17). The authors concluded that this difference was due to the surgeon learning curve. In our study, 31\% of patients had cardiopulmonary complications with TMM $\geq 2$ after VATS segmentectomy. This finding is a bit 
higher than in other studies (17-35\%) and is mainly due to the higher incidence of pneumonia (16-20). The higher incidence of pneumonia (17\%) in our study as compared to other studies (1.8-8.6\%) (16-19) could be explained by the broad pneumonia diagnostic criteria we used. In our initial experience, the operating time and the complication rate did not decrease over the 3-year study period. This is probably due to the great initial experience of surgeons in VATS procedure. Significant risk factors for complications after segmentectomy include male gender or $\mathrm{FEV}_{1} \leq 60 \%$ (17). However, we found that COPD, active smoking and the number of smoking pack years were also significant risk factors for complications after segmentectomy. These risk factors were already reported in others series after pulmonary resection $(20,21)$.

Pulmonary segmentectomy in NSCLC patients remains controversial. It is currently considered appropriate for tumors $\leq 2 \mathrm{~cm}$ with no nodal involvement (1,22-24). Despite the limited experience with VATS segmentectomy at our two institutions and the relatively short follow-up period, the oncological outcome at 3 years (91\% of DFS) is very similar to what other studies have found and appears to be acceptable. In our study, we also showed that a nodule size $>2$ centimeters is associated with a higher risk of recurrence. However, the oncological safety of segmentectomy for early stage cancer will remain controversial until the definitive results of the two ongoing randomized trials (NCT00499330) and (JCOG0802/WJOG4607L) comparing VATS segmentectomy and lobectomy. In addition, other factors, such as the consolidation of the tumor seems to be important to assess whether segmentectomy is indicated and can explain the difference in survival between lobectomy and segmentectomy (25).

This study has some limitations. It is a retrospective study from two institutions, includes a small number of patients, and there is no control group. Because there were few of each type of complication, stratification to analyze risk factors for each one was not possible. In addition, we lacked data about complications during the operations. The number of events during the follow-up period is low and the follow-up period of 3 years was too short to reach conclusions about the oncological safety of the segmentectomies performed in our hospitals.

In conclusion, our retrospective study of two institutions' experience suggests that the clinical outcome after segmentectomy by VATS seems to be acceptable. Particular attention must be paid to the postoperative period in current smokers and those with high pack-years because they are at greater risk for complications. The benefit of VATS segmentectomy versus VATS lobectomy during the postoperative course remain unknown. Furthermore, VATS segmentectomy remains technically difficult and previous experience with VATS lobectomy is mandatory to minimize complications.

\section{Acknowledgements}

We especially thank Pamela Derish for her review of the article and her help.

\section{Footnote}

Conflicts of Interest: The authors have no conflicts of interest to declare.

Ethical Statement: The study was approved by the local ethics committees (IRB: 2017-01767).

\section{References}

1. Cao C, Chandrakumar D, Gupta S, et al. Could less be more?-A systematic review and meta-analysis of sublobar resections versus lobectomy for non-small cell lung cancer according to patient selection. Lung Cancer 2015;89:121-32.

2. Dai C, Shen J, Ren Y, et al. Choice of Surgical Procedure for Patients With Non-Small-Cell Lung Cancer $</=1 \mathrm{~cm}$ or $>1$ to $2 \mathrm{~cm}$ Among Lobectomy, Segmentectomy, and Wedge Resection: A Population-Based Study. J Clin Oncol 2016;34:3175-82.

3. Hou B, Deng XF, Zhou D, et al. Segmentectomy versus wedge resection for the treatment of high-risk operable patients with stage I non-small cell lung cancer: a metaanalysis. Ther Adv Respir Dis 2016;10:435-43.

4. Qiu C, Wang G, Xu J, et al. Sublobectomy versus lobectomy for stage I non-small cell lung cancer in the elderly. Int J Surg 2017;37:1-7.

5. Zhao ZR, Situ DR, Lau RWH, et al. Comparison of Segmentectomy and Lobectomy in Stage IA Adenocarcinomas. J Thorac Oncol 2017;12:890-6.

6. Smith CB, Wolf A, Mhango G, et al. Impact of Surgeon Volume on Outcomes of Older Stage I Lung Cancer Patients Treated via Video-assisted Thoracoscopic Surgery. Semin Thorac Cardiovasc Surg 2017;29:223-30.

7. Taioli E, Liu B, Nicastri DG, et al. Personal and hospital factors associated with limited surgical resection for lung cancer, in-hospital mortality and complications in $\mathrm{New}$ 
York State. J Surg Oncol 2017;116:471-81.

8. Guigard S, Triponez F, Bédat B, et al. Usefulness of nearinfrared angiography for identifying the intersegmental plane and vascular supply during video-assisted thoracoscopic segmentectomy. Interact Cardiovasc Thorac Surg 2017;25:703-9.

9. Karenovics W, Licker M, Ellenberger C, et al. Shortterm preoperative exercise therapy does not improve longterm outcome after lung cancer surgery: a randomized controlled study. Eur J Cardiothorac Surg 2017;52:47-54.

10. Ferguson ND, Fan E, Camporota L, et al. The Berlin definition of ARDS: an expanded rationale, justification, and supplementary material. Intensive Care Med 2012;38:1573-82.

11. Harada H, Okada M, Sakamoto T, et al. Functional advantage after radical segmentectomy versus lobectomy for lung cancer. Ann Thorac Surg 2005;80:2041-5.

12. Kashiwabara K, Sasaki J, Mori T, et al. Relationship between functional preservation after segmentectomy and volume-reduction effects after lobectomy in stage I nonsmall cell lung cancer patients with emphysema. J Thorac Oncol 2009;4:1111-6.

13. Suzuki H, Morimoto J, Mizobuchi T, et al. Does segmentectomy really preserve the pulmonary function better than lobectomy for patients with early-stage lung cancer? Surg Today 2017;47:463-9.

14. Yoshimoto K, Nomori H, Mori T, et al. Postoperative change in pulmonary function of the ipsilateral preserved lung after segmentectomy versus lobectomy. Eur J Cardiothorac Surg 2010;37:36-9.

15. Zhang Y, Gao Y. Effects of VATS Lobectomy, VATS Anatomic Segmentectomy, and Open Thoracotomy on Pulmonary Function of Patients with Non-small Cell Lung Cancer. Zhongguo Fei Ai Za Zhi 2016;19:700-4.

16. Berry MF, Onaitis MW, Tong BC, et al. A model for morbidity after lung resection in octogenarians. Eur J Cardiothorac Surg 2011;39:989-94.

Cite this article as: Bédat B, Abdelnour-Berchtold E, Krueger T, Perentes JY, Ris HB, Triponez F, Licker MJ, Karenovics W, Gonzalez M. Clinical outcome and risk factors for complications after pulmonary segmentectomy by video-assisted thoracoscopic surgery: results of an initial experience. J Thorac Dis 2018;10(8):5023-5029. doi: 10.21037/jtd.2018.07.42
17. Traibi A, Grigoroiu M, Boulitrop C, et al. Predictive factors for complications of anatomical pulmonary segmentectomies. Interact Cardiovasc Thorac Surg 2013;17:838-44.

18. Deng B, Cassivi SD, de Andrade M, et al., Clinical outcomes and changes in lung function after segmentectomy versus lobectomy for lung cancer cases. J Thorac Cardiovasc Surg 2014;148:1186-92.e3.

19. Ojanguren A, Gossot D, Seguin-Givelet S. Division of the intersegmental plane during thoracoscopic segmentectomy: is stapling an issue? J Thorac Dis 2016;8:2158-64.

20. Smith CB, Kale M, Mhango G, et al. Comparative outcomes of elderly stage I lung cancer patients treated with segmentectomy via video-assisted thoracoscopic surgery versus open resection. J Thorac Oncol 2014;9:383-9.

21. Licker M, Karenovics W, Diaper J, et al. Short-Term Preoperative High-Intensity Interval Training in Patients Awaiting Lung Cancer Surgery: A Randomized Controlled Trial. J Thorac Oncol 2017;12:323-33.

22. Bedetti B, Bertolaccini L, Rocco R, et al. Segmentectomy versus lobectomy for stage I non-small cell lung cancer: a systematic review and meta-analysis. J Thorac Dis 2017;9:1615-23.

23. Dziedzic R, Zurek W, Marjanski T, et al. Stage I nonsmall-cell lung cancer: long-term results of lobectomy versus sublobar resection from the Polish National Lung Cancer Registry. Eur J Cardiothorac Surg 2017;52:363-9.

24. Liu Q, Wang H, Zhou D, et al. Comparison of clinical outcomes after thoracoscopic sublobectomy versus lobectomy for Stage I nonsmall cell lung cancer: A metaanalysis. J Cancer Res Ther 2016;12:926-31.

25. Hattori A, Matsunaga T, Takamochi K, et al. Locoregional recurrence after segmentectomy for clinical-T1aNOM0 radiologically solid non-small-cell lung carcinoma. Eur J Cardiothorac Surg 2017;51:518-25. 